\title{
Discrete-time Loop Transfer Recovery for Systems with Nonminimum Phase Zeros and Time Delays* $\dagger$
}

\author{
ZHIHONG ZHANG $\ddagger$ and JAMES S. FREUDENBERG§ \\ An analysis of the effects of nonminimum phase zeros and delays on the \\ discrete-time loop transfer recovery procedure leads to a better under- \\ standing of the procedure.
}

Key Words-Discrete time systems; optimal control; nonminimum-phased systems; delays.

\begin{abstract}
The purpose of this paper is to study what happens when the discrete-time loop transfer recovery (LTR) procedure is applied to plants with nonminimum phase zeros and with uniform time delay in all channels Explicit expressions are given for the asymptotic behavior of the resulting sensitivity function and loop transfer function. These results yield a better understanding of the mechanism of the discrete-time loop transfer recovery procedure and the design limitations due to nonminimum phase zeros and time delays.
\end{abstract}

\section{INTRODUCTION}

SINCE THE SEMINAL work of Kwakernaak (1969) and Doyle and Stein $(1979,1981)$ the loop transfer recovery method has received a lot of attention and evolved as a formal design procedure (Stein and Athans, 1987). Recently, extension of the loop transfer recovery design technique to discrete-time systems has been studied by a number of researchers (Maciejowski, 1985; Ishihara and Takeda, 1896; Niemann and Sogaard-Andersen, 1988; Kinnaert and Peng, 1990). Motivation for such an interest can be seen as follows. Firstly, guaranteed feedback properties for the discrete-time linear quadratic optimal regulator or Kalman filter do exist (Safonov, 1980; Shaked, 1986; Anderson and Moore, 1990) although they are not as good as in

\footnotetext{
* Received 3 September 1991; revised 22 May 1992; received in final form 4 June 1992 . The original version of this paper was not presented at any IFAC meeting. This paper was recommended for publication in revised form by Associate Editor R. V. Patel under the direction of Editor H. Kwakernaak.

† This work was supported in part by National Science Foundation under Grant ECS-8857510. It was conducted while the first author was with the Department of Electrical Engineering and Computer Science, University of Michigan, Ann Arbor, MI 48109, U.S.A.

¥ Systems Engineering Center, General Motors Corporation, 1151 Crooks Road, Troy, MI 48084, U.S.A.

$\S$ Department of Electrical Engineering and Computer Science, The University of Michigan, Ann Arbor, MI 48109-2122, U.S.A.
}

the continuous-time case. Nevertheless, it is desirable to have a method of recovering these properties. Secondly, the loop transfer recovery procedure significantly simplifies the use of the LQG methodology. Knowing that it will be recovered in the LTR procedure, the designer can mainly concentrate on the design of the state feedback loop.

There are two types of observers for discrete-time systems: predicting observers and filtering observers (Franklin and Powell, 1980). The predicting observer is used when there is a significant computation time and the filtering observer is used when the computation time is negligible. The standard state feedback scheme is dual to the predicting observer, but not to the filtering observer. This implies that there is a fundamental difference between the problem of recovering state feedback loop properties at the plant input using a specially tuned Kalman filter (which is a filtering observer) and the problem of recovering the optimal observer loop properties at the plant output using a specially tuned LQ optimal regulator.

Maciejowski (1985) studies the problem of recovering state feedback properties at the plant output. He shows that if the plant is minimum phase and has no time delays and if the cheap control regulator is applied to the filtering observer, then the feedback loop of the observer can be recovered. He also observed that although it is generally impossible to have perfect recovery when the plant is nonminimum phase or when the predicting observer has to be used, a useful degree of recovery is often obtained. An interpretation of this phenomenon in terms of asymptotic eigenvalue locations was also provided in Maciejowski (1985). Due to the fact that sampling often introduces nonmini- 
mum phase zeros (Åström et al., 1984) that computation time is not always negligible, and that many plants contain time delays, it is important to understand what happens when the loop transfer recovery procedure is applied under these conditions. The problem of loop transfer recovery for a plant with delays has been studied in Kinnaert and Peng (1990) for minimum phase systems. We shall see that their results can be included in our framework as a special case.

In this paper, we shall use the same approach as in Zhang and Freudenberg (1990) to study the effect of nonminimum phase zeros and time delays upon the LTR procedure. In Zhang and Freudenberg (1990) we studied loop transfer recovery for continuous-time nonminimum phase plants. We first factorized the plant into a minimum phase part and an all-pass factor expressed in terms of plant nonminimum phase zeros and their associated directions, and used these factors to derive explicit expressions for the loop transfer function and sensitivity function resulting from the LTR procedure. Design interpretations were then obtained from those expressions. The results of Zhang and Freudenberg (1990) were derived for the problem of recovery at the plant input and could also be applied, via duality, to the problem of recovery at the plant output. Since duality is not so complete with discrete-time systems, we shall only consider here the problem of recovery at the plant output. There are several procedures to achieve the loop transfer recovery (e.g. Niemann and Sogaard-Andersen, 1988; Tsui, 1989; Saberi and Sannuti, 1990; Chen et al., 1991). Our discussion will focus on the one based on Riccati equations (Maciejowski, 1985).

The rest of the paper is organized as follows. Section 2 contains definitions, some properties of nonminimum phase systems and a generalization of the minimum phase/all-pass factorization formulas in Enns (1984) and Zhang and Freudenberg (1990) to discrete-time systems. In Section 3, we derive explicit expressions for the limiting values of the sensitivity and loop transfer functions when the LTR procedure proposed in Maciejowski (1985) is applied to a nonminimum phase plant. Interpretations of the results are also given. The results of applying the LTR procedure with the predicting, rather than the filtering, observer are discussed in Section 4. In Section 5 we study the application of the LTR procedure to a plant with uniform time delay in all channels. An example is given in Section 6 to illustrate our results and conclusions are found in Section 7. An abbreviated version of this paper was presented in Zhang and Freudenberg (1991).
2. NOTATION AND ALL-PASS FACTORIZATION

In the following, we consider a discrete-time system described by state equations

$$
\begin{gathered}
x_{k+1}=A x_{k}+B u_{k}, \\
y_{k}=C x_{k},
\end{gathered}
$$

where $x \in R^{n}$ is the state, $u \in R^{m}$ is the control input, and $y \in R^{m}$ is the measured output. It is assumed that $(C, A)$ is observable, $(A, B)$ is controllable, $C$ and $B$ are each full rank, and $G(z):=C(z I-A)^{-1} B$. We shall use superscripts $T$ and $H$ to denote transpose and complex conjugate transpose, respectively. Notation $\bar{a}$ denotes complex conjugate of a complex number a.

Consider the state feedback control law

$$
u_{k}=-K_{c} x_{k} .
$$

Let $K_{c}$ be obtained by using the LQ optimization technique with the performance index

$$
J=\sum_{k=0}^{\infty}\left(u_{k}^{T} R u_{k}+x_{k}^{T} Q x_{k}\right),
$$

where $R>0$ and $Q=C^{T} C$. Then it is known that $K_{c}$ is given by

$$
K_{c}=\left(R+B^{T} M B\right)^{-1} B^{T} M A,
$$

where $M$ is the positive definite solution of the Riccati equation

$$
\begin{aligned}
M= & A^{T} M A-A^{T} M B\left(R+B^{T} M B\right)^{-1} \\
& \times B^{T} M A+Q .
\end{aligned}
$$

Define the loop transfer function of the optimal regulator loop

$$
\begin{aligned}
H_{s f}(z)= & K_{c}(z I-A)^{-1} B=\left(R+B^{T} M B\right)^{-1} \\
& \times B^{T} M A(z I-A)^{-1} B .
\end{aligned}
$$

The above control law assumes that all states are available for feedback. Typically, not all the states are measurable, and the missing states must be estimated from the output measurements $y_{l}, l \leq k$. There are two versions of full order estimates for the state $x_{k}$ : the filter estimate $\hat{x}_{k / k}$ which is based on measurements up to and including the current measurement $y_{k}$, and the predictor estimate $\hat{x}_{k / k-1}$ which is based on measurements up to $y_{k-1}$. Two observers commonly used in discrete-time systems are described as follows (Maciejowski, 1985; Franklin and Powell, 1980).

\subsection{Predicting observer}

The predicting observer is described by

$$
\hat{x}_{k+1 / k}=A \hat{x}_{k / k-1}+B u_{k}+K_{p}\left(y_{k}-C \hat{x}_{k / k-1}\right) \text {, }
$$

where the observer gain $K_{p}$ is chosen so that $A-K_{p} C$ is stable. When the predicting observer 
is used, the control law (3) is replaced by

$$
u_{k}=-K_{c} \hat{x}_{k / k-1} \text {. }
$$

This results in the predicting compensator

$$
U(z)=-F_{p}(z) Y(z),
$$

where

$$
F_{p}(z):=K_{c}\left[z I-A+B K_{c}+K_{p} C\right]^{-1} K_{p} .
$$

The predicting compensator is appropriate when the computation time is not negligible, and we cannot use the current measurement of the output to update the control.

\subsection{Filtering observer}

The filtering observer is described by

$$
\begin{gathered}
\hat{x}_{k+1 / k}=A \hat{x}_{k / k-1}+B u_{k}+A K_{f}\left(y_{k}-C \hat{x}_{k / k-1}\right), \\
\hat{x}_{k / k}=\hat{x}_{k / k-1}+K_{f}\left(y_{k}-C \hat{x}_{k / k-1}\right),
\end{gathered}
$$

where the observer gain $K_{f}$ is chosen such that $A-A K_{f} C$ is stable. In this case, the control law (3) is replaced by

$$
u_{k}=-K_{c} \hat{x}_{k / k} .
$$

This results in the filtering compensator

$$
U(z)=-F_{f}(z) Y(z),
$$

where

$$
F_{f}(z):=z K_{c}\left[z I-\left(I-K_{f} C\right)\left(A-B K_{c}\right)\right]^{-1} K_{f} .
$$

The filtering compensator is appropriate when the computation time is negligible, and we can use the current measurement of the output to update the control.

When equation (12) is used alone, we call it the 'predicting' version of the filtering observer, since it has the same form as a predicting observer with gain $K_{p}=A K_{f}$.

The Kalman filter is a filtering observer with

$$
K_{f}=P C^{T}\left(C P C^{T}+V\right)^{-1},
$$

where $P$ is the unique positive semidefinite solution to the dual algebraic Riccati equation

$$
P=A P A^{T}-A P C^{T}\left(C P C^{T}+V\right)^{-1} C P A^{T}+W,
$$

and $W \geqq 0$ and $V>0$ are fictitious process and measurement noise covariance matrices, respectively. Define the loop transfer function of the Kalman filter loop (see Fig. 1)

$$
\begin{aligned}
H_{\mathrm{ob}}(z)= & C(z I-A)^{-1} A K_{f}=C(z I-A)^{-1} \\
& \times A P C^{T}\left(C P C^{T}+V\right)^{-1} .
\end{aligned}
$$

Note that the optimal state feedback described by (1)-(6) and the optimal observer described by (12)-(13) and (17)-(18) are not dual, but that the optimal state feedback loop transfer function $H_{\mathrm{sf}}(z)$ and the optimal observer loop transfer function $H_{\mathrm{ob}}(z)$ are dual. Hence, the optimal state feedback is dual to the 'predicting' version of the optimal observer described by (12) and (17)-(18)

Guaranteed feedback properties of the discrete-time optimal regulator and Kalman filter loops also exist (Safonov, 1980; Shaked, 1986; Anderson and Moore, 1990). These properties can be derived from the discrete-time Kalman equality (or its dual)

$$
\begin{aligned}
& {\left[I+B^{T} \Phi\left(z^{-1}\right)^{T} K_{c}^{T}\right]\left(B^{T} M B+R\right)\left[I+K_{c} \Phi(z) B\right]} \\
& \quad=R+B^{T} \Phi\left(z^{-1}\right)^{T} Q \Phi(z) B,
\end{aligned}
$$

where $\Phi(z):=(z I-A)^{-1}$. These guaranteed properties are not as good as in the continuous case. In particular, there is no infinite gain margin. Of course, this makes sense because the zero at infinity is outside the unit circle and thus in the unstable region.

We now introduce some notation. First, let us define the following transfer function matrices. Observer loop transfer function: $H(z)=C(z I-$ $A)^{-1} A K_{f}$.

Observer sensitivity function: $S_{\mathrm{ob}}(z)=[I+$ $H(z)]^{-1}$.

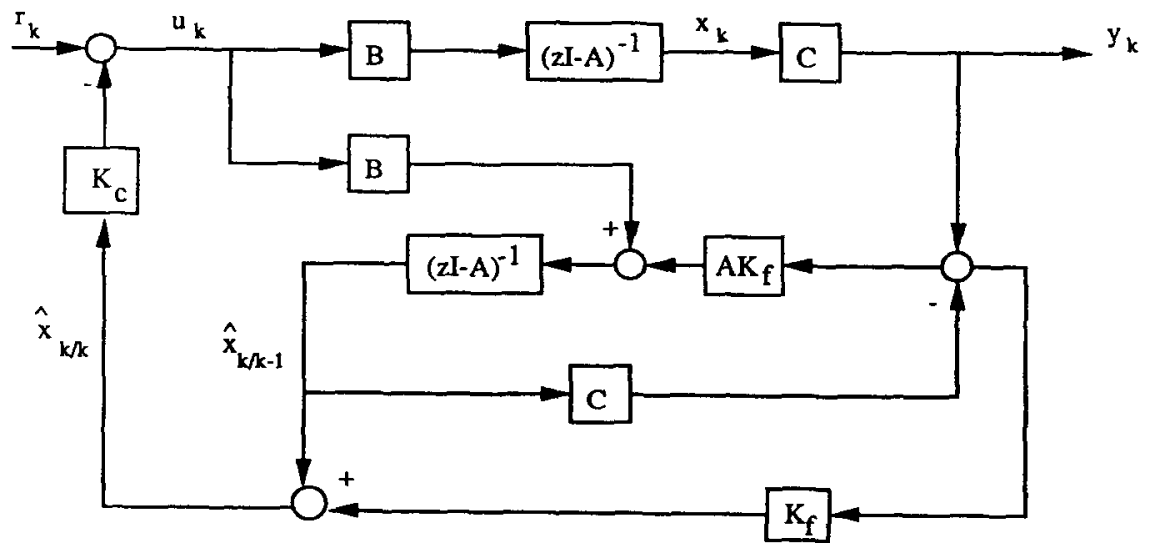

FIG. 1. System structure for the discrete-time loop transfer recovery. 
Output feedback loop transfer function: $L(z)=$ $G(z) F(z)$.

Output feedback sensitivity function: $S_{\text {out }}(z)=$ $[I+L(z)]^{-1}$.

$F(z)=F_{f}(z)$ or $F_{p}(z)$, depending upon which observer-based compensator is used. The following definitions are also needed in the subsequent development.

Definition 2.1 (Davison and Wang, 1974). The transmission zeros of system (1)-(2) are defined to be the set of complex numbers $a$ which satisfy the following inequality

$$
\operatorname{rank}\left[\begin{array}{cc}
a I-A & -B \\
-C & 0
\end{array}\right]<n+m .
$$

The multiplicity of $a$ is equal to its algebraic multiplicity as defined in MacFarlane and Karcanias (1976).

Definition 2.2 (MacFarlane and Karcanias, 1976). Let $a$ be a transmission zero of $G(z)$, so that

$$
\left[\begin{array}{ll}
x^{T} & w^{T}
\end{array}\right]\left[\begin{array}{cc}
a I-A & -B \\
-C & 0
\end{array}\right]=0,
$$

has a solution with $w^{H} w=1$. Then $x$ is called the left state zero direction and $w$ is called the output zero direction.

Definition 2.3. The system (1)-(2) is said to be nonminimum phase if at least one of its transmission zeros is outside the closed unit disk in the complex plane and such zeros are called nonminimum phase zeros of the system. Otherwise the system (1)-(2) is said to be minimum phase.

Note that we call a system minimum phase if it has no finite zeros outside the unit circle.

It is well known that nonminimum phase zeros of a transfer function $G(z)$ may be collected into a stable all-pass factor, i.e. $G(z)$ may be written as

$$
G(z)=C_{a}(z) G_{\mathrm{m}}(z),
$$

where $C_{a}(z)$ is stable, has zeros coinciding with the nonminimum phase zeros of $G(z)$, and satisfies $C_{a}\left(z^{-1}\right)^{T} C_{a}(z)=I$. The transfer function $G_{m}(z)$ is minimum phase and is termed the minimum phase counterpart of $G(z)$. The following lemma gives a constructive procedure for performing this factorization.

Lemma 2.1. Given the transfer function $G(z)=$ $C(z I-A)^{-1} B$ that has $l$ nonminimum phase zeros $a_{1}, a_{2}, \ldots, a_{l}$, (including multiplicities), the factorization (23) can be obtained using the following iterative procedure.
Factor out the nonminimum phase zeros of $G(z)$ one at a time as follows:

$$
\begin{aligned}
G(z)= & C_{a_{1}}(z) G_{m}^{1}(z), \\
G_{m}^{1}(z)= & C_{a_{2}}(z) G_{m}^{2}(z), \\
& \cdots, \\
G_{m}^{l-1}(z)= & C_{a_{l}}(z) G_{m}^{l}(z),
\end{aligned}
$$

where $G_{m}^{i}(z):=C_{m}^{i}(z I-A)^{-1} B, C_{m}^{0}:=C$, and for $i=1,2, \ldots, l$,

$$
\begin{gathered}
C_{a_{i}}(z)=I-\left(\frac{a_{i} \bar{a}_{i}-1}{a_{i}+1}\right)\left(\frac{z+1}{z \bar{a}_{i}-1}\right) \bar{\eta}_{i} \eta_{i}^{T}, \\
C_{m}^{i}=C_{m}^{i-1}-\left(\frac{a_{i} \bar{a}_{i}-1}{\bar{a}_{i}+1}\right) \bar{\eta}_{i} \xi_{i}^{T}(A+I) .
\end{gathered}
$$

The vectors $\eta_{i}$ and $\xi_{i}$ are solutions of

$$
\left[\begin{array}{ll}
\xi_{i}^{T} & \left.\eta_{i}^{T}\right]
\end{array}\right]\left[\begin{array}{cc}
a_{i} I-A & -B \\
-C_{m}^{i-1} & 0
\end{array}\right]=0,
$$

with $\eta_{i}^{H} \eta_{i}=1$. Then the factorization (23) is given by $G_{m}(z)=G_{m}^{I}(z)$ and $C_{a}(z)=$ $C_{a_{1}}(z) C_{a_{2}}(z) \cdots C_{a_{l}}(z)$.

Proof. Straightforward by applying a bilinear transformation to the continuous-time results of Zhang and Freudenberg (1990). Details can be found in Appendix B of Zhang (1990).

The above procedure yields a formula for the minimum-phase/all-pass factorization which is useful for our purpose. Other expressions for this factorization include the standard innerouter factorization (e.g. Francis, 1987) and a factorization due to Shaked (1990).

From the above lemma we can see that for a given transfer function $G(z)=C(z I-A)^{-1} B$ there always exist $C_{m}$ and $C_{a}(z)$ such that (23) can be written in the following form:

$$
G(z)=C_{a}(z) C_{m}(z I-A)^{-1} B .
$$

We shall now calculate the limiting value of the optimal regulator gain as the control cost approaches zero.

Theorem 2.1. Consider a nonminimum phase system $(A, B, C)$ and its minimum phase counterpart $\left(A, B, C_{m}\right)$, with $C_{m}$ as calculated in Lemma 2.1. Let $K_{c}$ be the feedback gain calculated according to (5) and (6) with $R=\left(1 / q^{2}\right) I$. Suppose that $C B=C A B=\cdots=$ $C A^{l-2} B=0$ and $\operatorname{det}\left(C A^{l-1} B\right) \neq 0$. Then $K_{c} \rightarrow$ $\left(C_{m} A^{l-1} B\right)^{-1} C_{m} A^{\prime}$ as $q \rightarrow \infty$.

Proof. Use duality and Theorem 3.1 of Shaked (1985). Note that since $C_{\mathrm{a}}(z)$ is bicausal, the structure of $G(z)$ at infinity is the same as that of $G_{m}(z)$ which implies that the conditions $C B=C A B=\cdots=C A^{l-2} B=0, \operatorname{det}\left(C A^{l-1} B\right) \neq$ 
0 are equivalent to the conditions $C_{m} B=$ $C_{m} A B=\cdots=C_{m} A^{l-2} B=0, \operatorname{det}\left(C_{m} A^{l-1} B\right) \neq 0$. Also note that the assumptions in Shaked (1985) that $A$ is stable and that the system $(A, B, C)$ has no poles or zeros at the origin can be removed (for details, see Appendix A of Zhang (1990)).

\section{LOOP TRANSFER RECOVERY WITH NONMINIMUM PHASE ZEROS}

The development of this section is based on the discrete-time loop transfer recovery procedure proposed by Maciejowski (1985). We first briefly state the procedure. Consider the observer-based output feedback system shown in Fig. 1. Suppose that the plant transfer matrix is minimum phase and $\operatorname{det}(C B) \neq 0$. The procedure is to design the observer loop $H(z)=$ $C(z I-A)^{-1} A K_{f}$ to meet design specifications (with augmented dynamics if necessary) and then to recover this loop asymptotically by tuning the state feedback gain $K_{c}$ which is obtained from (5) and (6) with $R=\left(1 / q^{2}\right) I$. If the filtering compensator $F_{f}(z)$ is used, then perfect recovery can be obtained asymptotically at the plant output, i.e. as $q \rightarrow \infty$,

$$
\begin{gathered}
G(z) F_{f}(z) \rightarrow H(z), \\
S_{\mathrm{out}}(z) \rightarrow S_{\mathrm{ob}}(z) .
\end{gathered}
$$

Here, as in the sequel, convergence of transfer functions is pointwise in $z$.

There are three crucial assumptions in the above LTR procedure, namely, the requirements that the plant is minimum phase, that $\operatorname{det}(C B) \neq 0$, and that the filtering compensator is used. We study next what happens when the minimum phase requirement is not satisfied. The use of the predicting compensator and the case where $\operatorname{det}(C B)=0$ will be discussed in the subsequent sections. The following theorem reveals the asymptotic behavior of the loop transfer and sensitivity functions when the plant is nonminimum phase.

Theorem 3.1. Suppose the plant $G(z)$ is factored as in (27), $\operatorname{det}(C B) \neq 0$, and the LTR procedure is applied using the filtering compensator $F_{f}(z)$. Then, as $q \rightarrow \infty$, the asymptotic behavior of the filtering compensator is given by

$$
\begin{aligned}
F_{f}(z) & \rightarrow\left(C_{m} \Phi(z) B\right)^{-1} \\
& \times\left[I-H_{m}(z)(I+H(z))^{-1} C_{a}(z)\right]^{-1} \\
& \times H_{m}(z)(I+H(z))^{-1},
\end{aligned}
$$

where $\quad H_{m}(z):=C_{m}(z I-A)^{-1} A K_{f}$. Consequently, the asymptotic values of the loop transfer and the sensitivity functions are given by

$$
L(z) \rightarrow[H(z)-E(z)][I+E(z)]^{-1},
$$

$$
S_{\mathrm{out}}(z) \rightarrow[I+E(z)] S_{\mathrm{ob}}(z),
$$

where $E(z):=\left[C-C_{a}(z) C_{m}\right](z I-A)^{-1} A K_{f}$ is called the error function.

\section{Proof. See Appendix A.}

Clearly, when the plant is minimum phase, (31) and (32) reduce to the known results (28) and (29), and the recovery is seen to be perfect. If the plant is nonminimum phase, on the other hand, then the quality of recovery at a given frequency depends upon the size of the error function $E(z)$ at that frequency. An inspection of the error function reveals that if, in addition to the assumptions of Theorem 3.1, the observer loop has the same nonminimum phase zero structure as the plant, i.e. if

$$
H(z)=C_{a}(z) C_{m}(z I-A)^{-1} A K_{f},
$$

then perfect recovery can be obtained. Also one can see from (30) that, in the minimum phase, case, the compensator constructs an inverse of the plant and substitutes the observer loop and that, in the nonminimum phase case, an inverse of the minimum phase counterpart of the plant is constructed. This provides a clear picture of how the recovery process works and complements the pole-zero cancellation explanation given by Maciejowski (1985). Next, we give a formula for calculating the error function $E(z)$ in terms of the nonminimum phase zeros and their associated directions.

Lemma 3.1. Let $G(z)$ have $l$ nonminimum phase zeros $a_{1}, a_{2}, \ldots, a_{l}$. Define $C_{a}^{0}(z)=I$, $C_{a}^{k}(z)=C_{a_{1}}(z) C_{a_{2}}(z) \cdots C_{a_{k}}(z), \quad E^{0}(z)=0$, and $E^{k}(z)=\left(C-C_{a}^{k}(z) C_{m}^{k}\right)(z I-A)^{-1} A K_{f}$ for $k=$ $1,2, \ldots, l$. Then

$$
\begin{aligned}
E(z)= & E^{\prime}(z)=\sum_{k=1}^{l} \frac{a_{k} \bar{a}_{k}-1}{z \bar{a}_{k}-1} \\
& \times C_{a}^{k-1}(z) \bar{\eta}_{k} \xi_{k}^{T} A K_{f},
\end{aligned}
$$

with $C_{m}^{k}$ and $C_{a_{k}}(z)$ defined by (24) and (25), and $\xi_{k}$ and $\eta_{k}$ defined by (26).

\section{Proof. See Appendix B.}

The following theorem states that perfect recovery can in fact be obtained in output directions that are orthogonal to those associated with the nonminimum phase zeros.

Theorem 3.2. Suppose that plant $G(z)$ has $l$ distinct nonminimum phase zeros $a_{1}, a_{2}, \ldots, a_{l}$ and that $\operatorname{det}(C B) \neq 0$. Let $w_{1}, w_{2}, \ldots, w_{l}$ denote the corresponding output zero directions defined by (22). Define $W$ to be the subspace of 
$C^{m}$ spanned by $w_{1}, w_{2}, \ldots, w_{l}$, and $W^{\perp}$ to be its orthogonal complement in $C^{m}$. Let $P_{W^{+}}$be a projection onto $W^{\perp}$. Then if the LTR procedure is applied using the filtering compensator $F_{f}(z)$, we have

$$
P_{W^{\perp}}^{T} S_{\text {out }}(z) \rightarrow P_{W^{1}}^{T} S_{o b}(z),
$$

as $q \rightarrow \infty$.

\section{Proof. See Appendix C.}

An analogous result may be obtained for the dual version of the continuous-time recovery result in Zhang and Freudenberg (1990).

For systems that have a single (real) nonminimum phase zero, more insightful expressions can be obtained.

Corollary 3.1. Consider a plant $G(z)$ that has only one nonminimum phase zero, and assume it is at $z=a$. Suppose that $\operatorname{det}(C B) \neq 0$ and that the LTR procedure is applied using the filtering compensator $F_{f}(z)$. Then the sensitivity function of the system satisfies

$$
S_{\mathrm{out}}(z) \rightarrow\left[I+\frac{a^{2}-1}{z a-1} w w^{T} H(a)\right] S_{o b}(z),
$$

as $q \rightarrow \infty$, where $w^{T} H(a)=w^{T} C(a I-A)^{-1} A K_{f}$ and $w$ is the output zero direction determined by (22). If the plant is scalar, then

$$
S_{\mathrm{out}}(z) \rightarrow\left[1+\frac{a^{2}-1}{s a-1} H(a)\right] S_{o b}(z),
$$

as $q \rightarrow \infty$.

Proof. It follows from Lemma 3.1 by setting $l=1$ that

$$
E(z)=\frac{a^{2}-1}{z a-1} \eta_{1} \xi_{1}^{T} A K_{f} .
$$

The limit (35) follows by noting $\xi_{1}^{T} A K_{f}=$ $\eta_{1}^{T} C(a I-A)^{-1} A K_{f}$ and $\eta_{1}=w$. For scalar systems, $w=1$. Hence (35) reduces to (36).

One can see that if the target feedback loop $H(z)$ also has a zero at $z=a$, with output zero direction $w$, then perfect recovery is possible. Otherwise there will be an unavoidable error in recovery, whose size depends upon the value of $\left\|w^{T} H(a)\right\|$, and thus upon the location of the zero relative to the frequency range over which the target feedback loop gain is large in the direction $w$. It is clear from the above results that if the nonminimum phase zero is far outside the bandwidth of the target feedback loop, then good recovery can be obtained. This confirms the observation by Maciejowski (1985). Also, there appears to be a tradeoff between the feedback properties of the target feedback loop and the quality of recovery. Further quantifications of this tradeoff may be performed using Poisson integral relations similarly to the continuous case (Zhang and Freudenberg, 1990). This has recently been done by Léon de la Barra (1991).

\section{USE OF THE PREDICTING COMPENSATOR}

The recovery procedure in the previous section assumes negligible computation time, so that it is possible to implement the filtering compensator. This may be impractical, since the time required to compute the control signal is not always negligible. If this is the case, then the feedback law (9) has to be used, resulting in the predicting compensator $F_{p}(z)$ with $K_{p}=A K_{f}$. Although it has been shown (Maciejowski, 1985) that in this case perfect recovery cannot generally be obtained, it is of interest to investigate what happens if we try to apply the recovery procedure using the predicting compensator.

Theorem 4.1. Suppose that the plant $G(z)$ is factored as in (27), that $\operatorname{det}(C B) \neq 0$, and that the LTR procedure is applied using the predicting compensator $F_{p}(z)$ with $K_{p}=A K_{f}$. Then, as $q \rightarrow \infty$, the asymptotic values of the loop transfer and the sensitivity functions are given by

$$
\begin{gathered}
L(z) \rightarrow\left[H(z)-E_{p}(z)\right]\left[I+E_{p}(z)\right]^{-1}, \\
S_{\mathrm{our}}(z) \rightarrow\left[I+E_{p}(z)\right] S_{\mathrm{ob}}(z),
\end{gathered}
$$

where the error function is given by

$$
\begin{aligned}
E_{p}(z):= & z^{-1}\left[z C-C_{a}(z) C_{m} A\right] \\
& \times(z I-A)^{-1} A K_{f} .
\end{aligned}
$$

Proof. Similar to proof of Theorem 3.1, straightforward calculation of $G(z) F_{p}(z)$ by substituting $\left(C_{m} B\right)^{-1} C_{m} A$ for $K_{c}$.

The error function $E_{p}(z)$ can be calculated using the formulas for $C_{a}(z)$ and $C_{m}$ that we developed in Section 2. Note that the above result still holds without assuming $K_{p}=A K_{f}$, as long as the target observer loop $H(z)$ is interpreted as $C(z I-A)^{-1} K_{p}$ in such a case.

Corollary 4.1. Suppose, in addition to the assumptions of Theorem 4.1, that the plant $G(z)$ is minimum phase. Then the error function $E_{p}(z)$ reduces to

$$
E_{p}(z)=z^{-1} C A K_{f}
$$


It follows that if the observer gain satisfies

$$
C A K_{f}=0 \text {, }
$$

then perfect recovery can be obtained.

As noted by Maciejowski (1985), perfect recovery is generally unattainable with the predicting compensator even for minimum phase plants. The difference between the results obtained in this case for the two compensators $F_{f}(z)$ and $F_{p}(z)$ can be explained as follows. Note first that the assumption $\operatorname{det}(C B) \neq 0$ implies that the plant has an inherent one-step delay in all channels. Suppose that the observer loop also has a one-step delay. Then the only way that this loop can be perfectly recovered by the loop with observer-based compensator is for this compensator to have a direct feedthrough from the plant output to the control input, i.e. to have a proper inverse. An inspection of (11), (16), and Fig. 1 reveals that this is possible only if the filtering compensator is implemented. On the other hand, suppose that the observer gain satisfies (41), so that the observer loop has (at least) an inherent two-step delay. Then it becomes potentially possible for the observer loop to be recovered using the predicting compensator. Unfortunately, requiring such a two-step delay would result in a less satisfactory observer loop than would be the case if a shorter delay were present. This situation is analogous to that studied in the previous section, where we saw that only nonminimum phase (and therefore inferior) observer loops could be recovered. Hence, we see that when the computation time is not negligible and the predicting compensator has to be used, perfect recovery is possible only if the target observer loop is constrained to have an extra step delay. This observation is consistent with that of Ishihara and Takeda (1986).

It is of interest to study how recovery takes place when the filtering compensator is implemented to recover an observer loop with (at least) a two-step delay in all channels. From the above discussion, we know that such an observer loop satisfies $C A K_{f}=0$. Since the asymptotic control gain satisfies $K_{c} \rightarrow$ $(C B)^{-1} C A$, it follows that the condition $C A K_{f}=0$ implies that $K_{c} K_{f} \rightarrow 0$. Therefore, as $q \rightarrow \infty$, the feedback control law

$$
\begin{aligned}
u_{k}=-K_{c} \hat{x}_{k / k}= & -K_{c}\left[\hat{x}_{k / k-1}+K_{f}\left(y_{k}-C \hat{x}_{k / k-1}\right)\right] \\
& \rightarrow-K_{c} \hat{x}_{k / k-1} .
\end{aligned}
$$

Hence, asymptotically the optimal state feedback control law does not utilize those states that are updated using the current output measurement. Therefore the direct feedthrough link in Fig. 1 is not used.
The role of the assumption that $\operatorname{det}(C B) \neq 0$ is also now clear. If it is not satisfied, then the plant would have at least a two-step delay in some channels, and it would be impossible for the observer-based output feedback loop to asymptotically recover an observer loop with a one-step delay in all channels.

\section{LOOP TRANSFER RECOVERY WITH TIME DELAYS}

In the previous sections, the assumption is made that $\operatorname{det}(C B) \neq 0$. However, this assumption will be violated for systems with at least a two-step time delay in some channel of the plant. It has been observed (Maciejowski, 1985) that perfect recovery cannot generally be obtained in this case, but it would be of interest to see what happens when the LTR procedure is applied anyway. In the following, we consider application of the LTR procedure to a class of plants whose delay structure is characterized by

$$
C B=C A B=\cdots=C A^{l-2} B=0, \operatorname{det}\left(C A^{l-1} B\right) \neq 0,
$$

where $l \geq 2$, i.e. plants that have a uniforin $l$-step delay in all channels. This problem has been studied by Kinnaert and Peng (1990) for minimum phase systems using the predicting compensator in the LTR procedure. The following results extend and complement those of Kinnaert and Peng (1990).

Theorem 5.1. Suppose that the plant $G(z)$ is factored as in (27) and has a uniform $l$-step delay as characterized by (42). Assume that the LTR procedure is applied to the system using either the filtering compensator $F_{f}(z)$ or the predicting compensator $F_{p}(z)$ with $K_{p}=A K_{f}$. Then, as $q \rightarrow \infty$, the asymptotic values of the loop transfer and the sensitivity functions are given by

$$
\begin{gathered}
L(z) \rightarrow\left[H(z)-E_{d}(z)\right]\left[I+E_{d}(z)\right]^{-1}, \\
S_{\text {our }}(z) \rightarrow\left[I+E_{d}(z)\right] S_{o b}(z),
\end{gathered}
$$

where $H(z)=C(z I-A)^{-1} A K_{f}$. The error function $E_{d}(z)$ is given by

$$
\begin{aligned}
E_{d}(z)= & \left(C-\frac{1}{z^{l-1}} C_{a}(z) C_{m} A^{l-1}\right) \\
& \times(z I-A)^{-1} A K_{f},
\end{aligned}
$$

when the filtering compensator is used, and is given by

$$
\begin{aligned}
E_{d}(z)= & \left(C-\frac{1}{z^{\prime}} C_{a}(z) C_{m} A^{\prime}\right) \\
& \times(z I-A)^{-1} K_{p},
\end{aligned}
$$

when the predicting compensator with $K_{p}=A K_{f}$ is used. 
Proof. Direct calculation of the loop transfer function using the cheap control gain given in Theorem 2.1.

Notice that (46) also holds for a general predicting compensator with $K_{p} \neq A K_{f}$. However, the definition of $H(z)$ in (43) and (44) has to be changed to $H(z)=C(z I-A)^{-1} K_{p}$ in such a case. If the plant is minimum phase, then (46) reduces to

$$
E_{d}(z)=H(z)-\frac{1}{z^{l}} C A^{l}(z I-A)^{-1} K_{p} .
$$

Substituting the above into (43) results in the following limiting value of the loop transfer function

$$
\begin{aligned}
L(z)= & \frac{1}{z^{l}} C A^{l}(z I-A)^{-1} K_{p}[I+H(z) \\
& \left.-\frac{1}{z^{l}} C A^{l}(z I-A)^{-1} K_{p}\right]^{-1} \\
= & C(z I-A)^{-1} A^{l} K_{p}\left(z^{l} I+z^{l-1} C K_{p}\right. \\
& \left.+\cdots+C A^{l-1} K_{p}\right)^{-1}
\end{aligned}
$$

which gives the same result as Theorem 3 of Kinnaert and Peng (1990).

One can see from Theorem 5.1 that the recovery error $E_{d}(z)$ is a function of the nonminimum zeros, the time delays, and the observer loop gain $\left(K_{f}\right.$ or $\left.K_{p}\right)$ which implies that all those factors can affect the quality of recovery. For a given target observer loop, the recovery error can be calculated, a priori, using (45) or (46) and our formulas for $C_{a}(z)$ and $C_{m}$, to determine how much and at which frequencies it can be recovered. Since $E_{d}(z)$ is generally nonzero, perfect recovery cannot be obtained in general and the quality of recovery at a certain frequency will depend upon the size of the error function at that frequency. However, if the observer loop to be recovered meets certain constraints, then perfect recovery is possible. First, we consider the case where the filtering compensator is used. It follows from (45) that if the observer loop transfer function $H(z)=$ $C(z I-A)^{-1} A K_{f}$, i.e. the transfer function to be recovered, satisfies

(1) $H(z)=C_{a}(z) C_{m}(z I-A)^{-1} A K_{f}$,

(2) $C A K_{f}=C A^{2} K_{f}=\cdots=C A^{l-1} K_{f}=0$,

then the error function $E_{d}(z)$ will be identically zero. In other words, if the observer loop is chosen to have the same nonminimum phase structure and at least as many steps of time delay as the plant, then it can be perfectly recovered using the filtering compensator.

For the case where the general predicting compensator is used, if the observer loop $H(z)=C(z I-A)^{-1} K_{p}$ satisfies

(1) $H(z)=C_{a}(z) C_{m}(z I-A)^{-1} K_{p}$,

(2) $C K_{p}=C A K_{p}=\cdots=C A^{l-1} K_{p}=0$, then one can show that

$$
H(z)=\frac{1}{z^{l}} C_{a}(z) C_{m} A^{l}(z I-A)^{-1} K_{p},
$$

which, by (46), implies that the recovery error $E_{d}(z)$ is zero. Hence, to have perfect recovery using the predicting compensator, the target observer loop has to have the same nonminimum phase zero structure as the plant and have at least one more step of time delay than the plant.

From the above discussion, one can see that the nonminimum phase plant zeros, plant time delays, and controller computation delays all impose constraints upon the class of recoverable target loop transfer functions. Since the recovery error is a function of the total time delay in the feedback loop, it follows that time delays in the plant and controller have the same effect on the loop transfer recovery. This observation is consistent with that of Kinnaert and Peng (1990).

Finally, note that our results concerning LTR using the predicting compensator could also be applied, via duality, to the problem of recovery at the plant input.

\section{EXAMPLE}

To illustrate some of our results, let us apply the LTR procedure to a nonminimum phase plant. Consider a sampled data system composed of a zero-order hold, a linear time-invariant continuous system $G(s)=\frac{1}{(s+1)^{3}}$ and a sampler
in series (see Fig. 2).

The corresponding pulse transfer function with sampling period $T$ is given by Åström et al. (1984).

$$
G(z)=\frac{b_{1} z^{2}+b_{2} z+b_{3}}{\left(z-e^{-T}\right)^{3}}
$$

where

$$
b_{1}=1-\left(1+T+\frac{T^{2}}{2}\right) e^{-T},
$$



FIG. 2. Sampled data system. 


$$
\begin{gathered}
b_{2}=\left(-2+T+\frac{T^{2}}{2}\right) e^{-T}+\left(2+T-\frac{T^{2}}{2}\right) e^{-2 T}, \\
b_{3}=\left(1-T+\frac{T^{2}}{2}\right) e^{-2 T}-e^{-3 T} .
\end{gathered}
$$

This transfer function has a real nonminimum phase zero for $0<T<1.8399$. According to Åström et al. (1984), nonminimum phase sampling zeros usually lie near the negative real axis when the sampling period is sufficiently small. For this example, the nonminimum phase zero approaches -3.732 as the sampling period goes to zero. It follows from Corollary 3.1 that good recovery may be obtained for this example when the sampling period is small, since the nonminimum phase sampling zero is expected to be outside the bandwidth of the target observer loop. We shall see in the following that this is indeed the case.

A realization of $G(z)$ is given by

$$
\begin{gathered}
A=\left[\begin{array}{ccc}
0 & 1 & 0 \\
0 & 0 & 1 \\
e^{-3 T} & -3 e^{-2 T} & 3 e^{-T}
\end{array}\right], \quad B=\left[\begin{array}{l}
0 \\
0 \\
1
\end{array}\right], \\
C=\left[\begin{array}{lll}
b_{3} & b_{2} & b_{1}
\end{array}\right] .
\end{gathered}
$$

The observer loop is designed using (17)-(18) with weightings $V=1$ and $W=B B^{T}$. Results obtained by applying the LTR procedure with the filtering compensator are given in Figs 3-9. For the first set of figures (Figs 3-5), we choose the sampling period $T=0.05$ which results in a nonminimum phase zero at $z=-3.5949$. The plots demonstrate that the sensitivity function $S_{\text {out }}(z)$ indeed converges to the function we predicted in Theorem 3.1. The second set of figures (Figs 6-9) shows how the length of the sampling period affects the location of the nonminimum phase zero $z=a$ which, in turn, affects the quality of recovery. First, we notice that the nonminimum phase sampling zero moves away from the unit disk along the real negative axis as the sampling period decreases. This implies that the nonminimum phase sampling zero gets farther away from the bandwidth of the target loop transfer function as the sampling period gets smaller. In these figures we plot the recoverable sensitivity function (solid line) and the desired target sensitivity function (dashed line). As expected, we observe that the recoverable sensitivity function approaches the target sensitivity function as the sampling period decreases.

\section{CONCLUSIONS}

In this paper, we have studied applications of the discrete-time LTR procedure to plants with nonminimum phase zeros and time delays. Explicit expressions are derived for the asymptotic behavior of the sensitivity function and loop transfer function resulting from the LTR procedure. The results are given for both filtering compensator and predicting compensator cases. For a given target loop, these expressions show a priori, how much, at which frequencies and in what directions the loop can be recovered. From our results, we can see that the LTR procedure, if used properly, may still be an effective design approach for discrete-time systems with nonminimum phase zeros and time delays. The plant nonminimum phase zero and time delay structures and computation delays

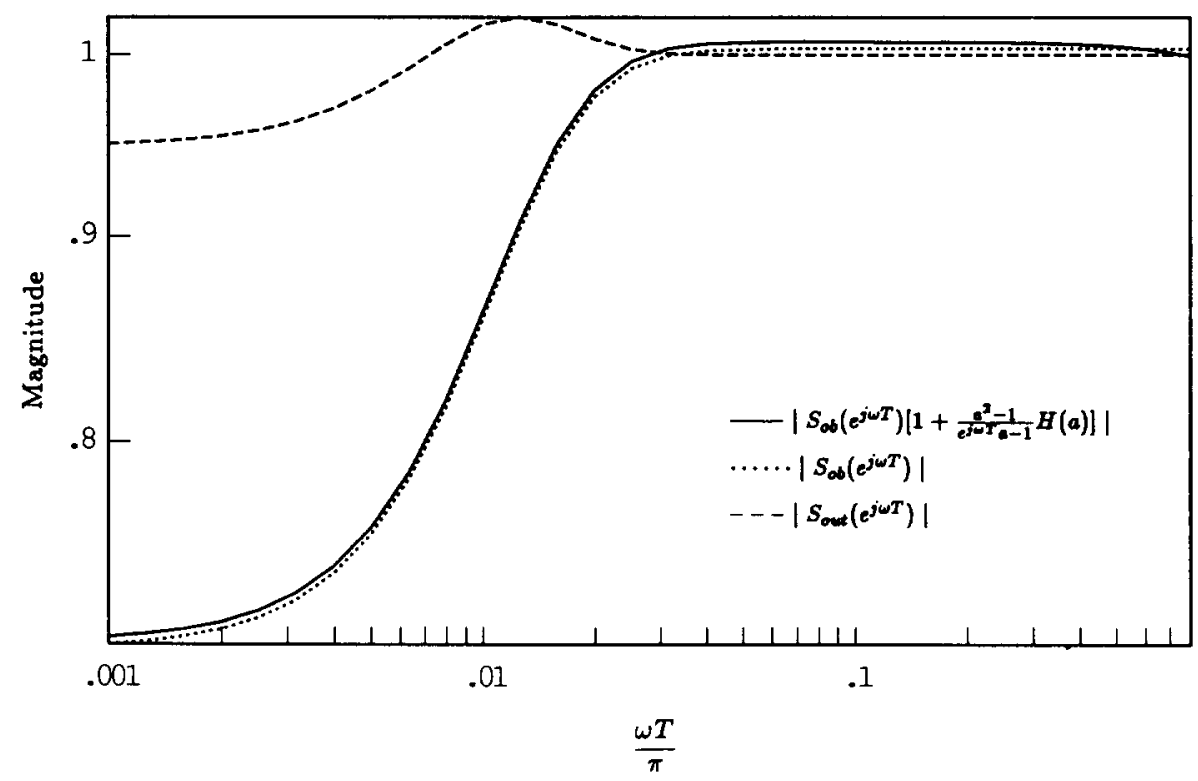

FIG. 3. Discrete-time asymptotic recovery: $q=1$. 


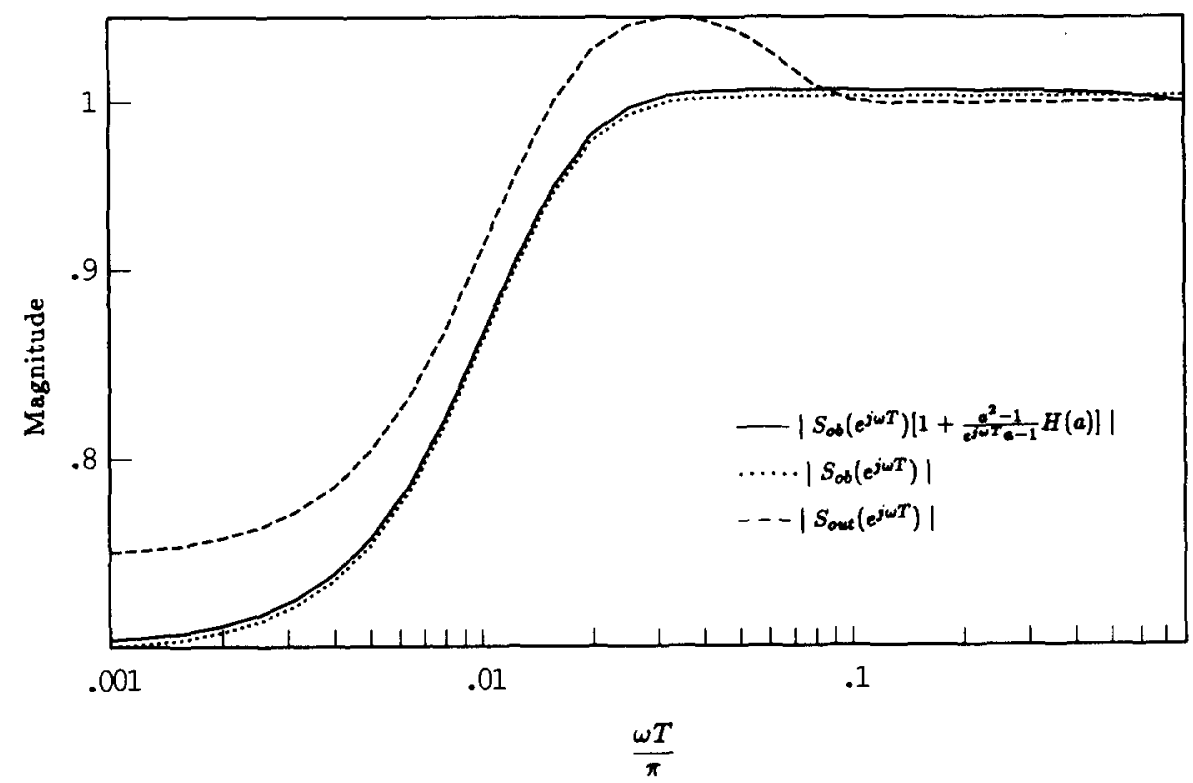

FIG. 4. Discrete-time asymptotic recovery: $q=100$.

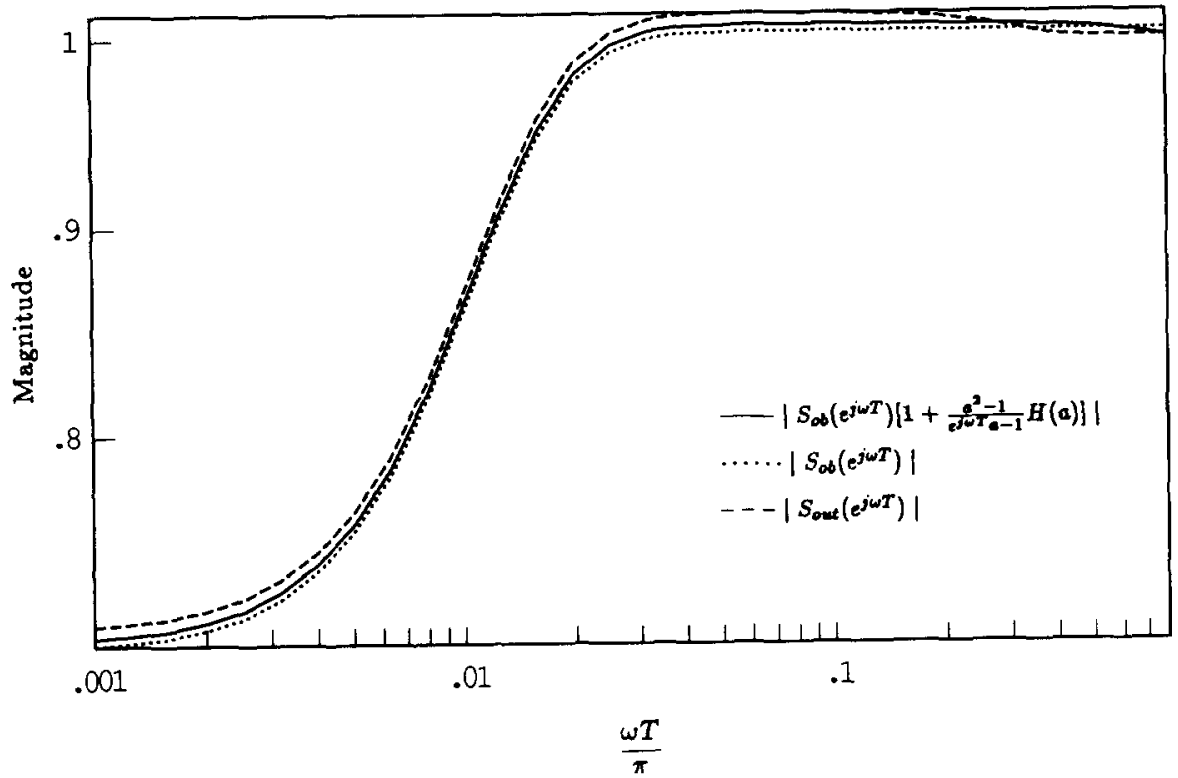

Fig. 5. Discrete-time asymptotic recovery: $q=10,000$.



FIG. 6. Discrete-time LTR vs sampling period: $T=0.5$ and $a=-2.5782$. 


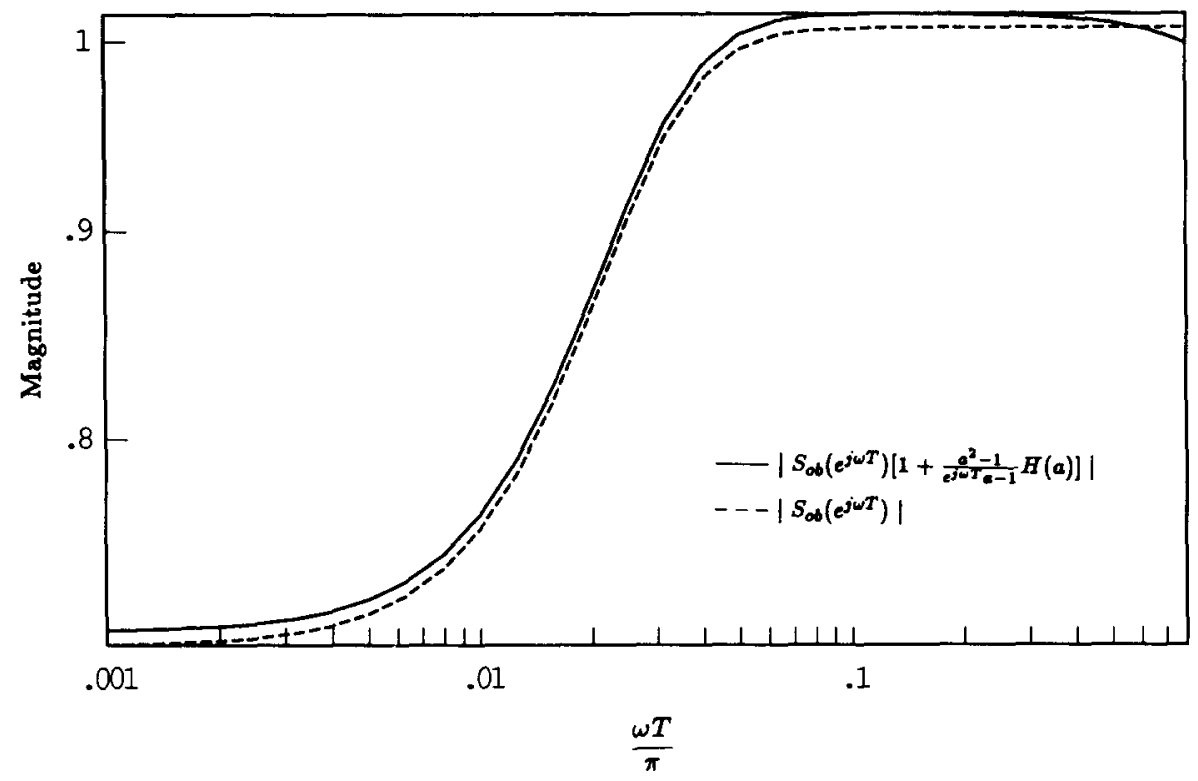

FIG. 7. Discrete-time LTR vs sampling period: $T=0.1$ and $a=-3.4631$.

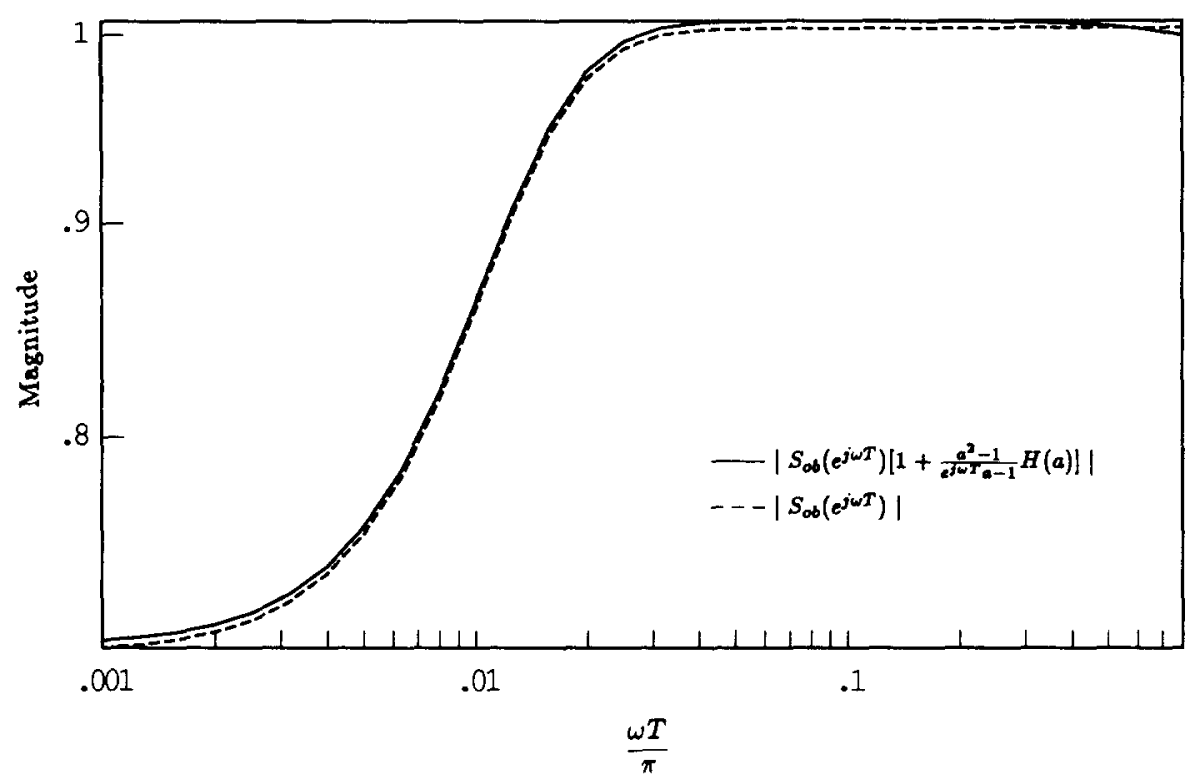

FIG. 8. Discrete-time LTR vs sampling period: $T=0.05$ and $a=-3.5949$.



FIG. 9. Discrete-time LTR vs sampling period: $T=0.01$ and $a=-3.7042$. 
essentially impose certain constraints upon the recoverable target loop transfer functions. In this paper, it is assumed that the plant is square and has uniform delay in all channels; further research is needed to extend the results to cases where those assumptions are not satisfied. The general results on singular discrete-time filtering problem by Shaked (1985) may prove useful in this regard.

\section{REFERENCES}

Anderson, B. and J. B. Moore (1990). Optimal Control: Linear Quadratic Methods. Prentice Hall, Englewood Cliffs, NJ.

Åström, K. J., P. Hagander and J. Sternby (1984). Zeros of sampled systems. Automatica, 20, 31-38.

Chen, B. M., A. Saberi and P. Sannuti (1991). A new stable compensator design for exact and approximate loop transfer recovery. Automatica, 27, 257-280.

Davison, E. J. and S. H. Wang (1974). Properties and calculation of transmission zeros of linear multivariable systems. Automatica, 10, 643-658.

Doyle, J. C. and G. Stein (1979). Robustness with observers. IEEE Trans. Aut. Control, AC-24, 607-611.

Doyle, J. C. and G. Stein (1981). Multivariable feedback design: Concepts for a classical/modern synthesis. IEEE Trans. Aut. Control, AC-26, 4-16.

Enns, D. (1984). Model reduction for control system design. $\mathrm{Ph}$. D. dissertation, Stanford University, Stanford, CA.

Francis, B. A. (1987). A Course in $\mathrm{H}_{\infty}$ Control Theory. Springer-Verlag, New York.

Franklin, G. and J. D. Powell (1980). Digital Control of Dynamic Systems. Addison-Wesley, New York.

Ishihara, T. and H. Takeda (1986). Loop transfer recovery techniques for discrete-time optimal regulators using prediction estimators. IEEE Trans. Aut. Control, AC-31, $1149-1151$.

Kinnaert, M. and Y. Peng (1990). Discrete-time LQG/LTR techniques for systems with time delays. Systems and Control Letters, 15, 303-311.

Kwakernaak, H. (1969). Optimal low-sensitivity linear feedback systems. Automatica, 5, 279-286.

Léon de la Barra, B. A. (1991). Frequency domain tradeoffs in loop transfer recovery for multivariable nonminimum phase discrete-time systems. Technical Report EE9117. University of Newcastle, Australia.

MacFarlane, A. G. J. and N. Karcanias (1976). Poles and zeros of linear multivariable systems: a survey of the algebraic, geometric and complex-variable theory. Int. J. Control, 24, 33-74.

Maciejowski, J. M. (1985). Asymptotic recovery for discrete-time systems. IEEE Trans. Aut. Control, AC-30, 602-605.

Niemann, H. H. and P. Sogaard-Andersen (1988). New results in discrete-time loop transfer recovery. Proc. of American Control Conf., pp. 2483-2489.

Saberi, A. and P. Sannuti (1990). Observer design for loop transfer recovery and for uncertain dynamical systems. IEEE Trans. Aut. Control, 35, 878-897.

Safonov, M. G. (1980). Stability and Robustness of Multivariable Feedback Systems. MIT Press, MA.

Shaked, U. (1985). Explicit solution to the singular discrete-time stationary linear filtering problem. IEEE Trans. Aut. Control, 30, 34-47.

Shaked, U. (1986). Guaranteed stability margin for the discrete-time linear quadratic optimal regulator. IEEE Trans. Aut. Control, AC-31, 162-165.

Shaked, U. (1990). An explicit expression for the minimum-phase image of transfer function matrices. IEEE Trans. Aut. Control, 34, 1290-1293.

Stein, G. and M. Athans (1987). The LQG/LTR procedure for multivariable feedback control design. IEEE Trans. Aut. Control, AC-32, 105-114.
Tsui, C. C. (1989). On loop transfer recovery. Proc. of American Contr. Conf., Pittsburgh, PA.

Zhang, Z. (1990). Loop transfer recovery for nonminimum phase plants and ill-conditioned plants. Ph.D. Thesis, The University of Michigan, Ann Arbor, MI.

Zhang, Z. and J. S. Freudenberg (1990). Loop transfer recovery for nonminimum phase plant. IEEE Trans. Aut. Control, AC-35, 547-553.

Zhang, Z. and J. S. Freudenberg (1991). On discrete-time loop transfer recovery. Proc. of American Contr. Conf., Boston, MA.

\section{APPENDIX A: PROOF OF THEOREM 3.1.}

By Theorem 2.1, we know that $K_{c} \rightarrow\left(C_{m} B\right)^{-1} C_{m} A$ as $q \rightarrow \infty$. Now evaluate the open loop transfer function $L(z)$ in the limit as $q \rightarrow \infty$. Define $\Phi(z)=(z I-A)^{-1}, F_{f}(z)$ can be rewritten as

$$
\begin{aligned}
F_{f}(z)= & z K_{c}\left[I+\Phi(z) B K_{c}+\Phi(z) K_{f} C\left(A-B K_{c}\right)\right]^{-1} \Phi(z) K_{f} \\
= & z K_{c}\left[I+\left(I+\Phi(z) K_{f} C A\right)^{-1} \Phi(z) B K_{c}\right. \\
& \left.-\left(I+\Phi(z) K_{f} C A\right)^{-1} \Phi(z) K_{f} C B K_{c}\right]^{-1} \\
& \times\left(I+\Phi(z) K_{f} C A\right)^{-1} \Phi(z) K_{f} .
\end{aligned}
$$

Note that $H_{m}(z)=C_{m} \Phi(z) A K_{f}$. Since $K_{c} \rightarrow\left(C_{m} B\right)^{-1} C_{m} A$ as $q \rightarrow \infty$, it follows that

$$
\begin{aligned}
F_{f}(z) \rightarrow & z\left(C_{m} B\right)^{-1} C_{m} A\left[I+\left(I+\Phi(z) K_{f} C A\right)^{-1} \Phi(z) B\right. \\
& \times\left(C_{m} B\right)^{-1} C_{m} A-\left(I+\Phi(z) K_{f} C A\right)^{-1} \Phi(z) K_{f} C B \\
& \left.\times\left(C_{m} B\right)^{-1} C_{m} A\right]^{-1}\left(I+\Phi(z) K_{f} C A\right)^{-1} \Phi(z) K_{f} \\
= & z\left(C_{m} B\right)^{-1}\left[I+C_{m} A\left(I+\Phi(z) K_{f} C A\right)^{-1} \Phi(z) B\left(C_{m} B\right)^{-1}\right. \\
& \left.-H_{m}(z)(I+H(z))^{-1} C B\left(C_{m} B\right)^{-1}\right]^{-1} H_{m}(z)(I+H(z))^{-1} \\
= & z\left[C_{m} B+C_{m} A\left(I-\Phi(z) K_{f} C A\left(I+\Phi(z) K_{f} C A\right)^{-1}\right) \Phi(z) B\right. \\
& \left.-H_{m}(z)(I+H(z))^{-1} C B\right]^{-1} H_{m}(z)(I+H(z))^{-1} \\
= & z\left[C_{m} B+C_{m} A \Phi(z) B-H_{m}(z)(I+H(z))^{-1} C A \Phi(z) B\right. \\
& \left.-H_{m}(z)(I+H(z))^{-1} C B\right]^{-1} H_{m}(z)(I+H(z))^{-1} \\
= & z\left[C_{m}(I+A \Phi(z)) B-H_{m}(z)(I+H(z))^{-1}\right. \\
& \times C(I+A \Phi(z)) B]^{-1} H_{m}(z)(I+H(z))^{-1} \\
= & {\left[C_{m} \Phi(z) B-H_{m}(z)(I+H(z))^{-1} C \Phi(z) B\right]^{-1} } \\
& \times H_{m}(z)(I+H(z))^{-1} .
\end{aligned}
$$

Note that $C \Phi(z) B=C_{a}(z) C_{m} \Phi(z) B$, we obtain that

$$
\begin{aligned}
F_{f}(z) \rightarrow & \left(C_{m} \Phi(z) B\right)^{-1}\left[I-H_{m}(z)(I+H(z))^{-1} C_{a}(z)\right]^{-1} \\
& \times H_{m}(z)(I+H(z))^{-1} .
\end{aligned}
$$

This completes the proof of (30). By definition of $E(z)$, we have that

$$
\begin{aligned}
L(z) \rightarrow & C \Phi(z) B\left(C_{m} \Phi(z) B\right)^{-1}\left[I-H_{m}(z)(I+H(z))^{-1}\right. \\
& \left.\times C_{a}(z)\right]^{-1} H_{m}(z)(I+H(z))^{-1} \\
= & C_{a}(z) H_{m}(z)\left[I+H(z)-C_{a}(z) H_{m}(z)\right]^{-1} \\
= & {[H(z)-E(z)][I+E(z)]^{-1}, }
\end{aligned}
$$

as $q \rightarrow \infty$. This gives (31). Since $S_{\text {out }}(z)=[I+L(z)]^{-1}$, (32) follows readily.

\section{APPENDIX B: PROOF OF LEMMA 3.1}

First, let us derive a recursive formula for $E^{k}(z)$. Using the factorization formulas (24) and (25), we get

$$
\begin{aligned}
C- & C_{a}^{k}(z) C_{m}^{k} \\
= & C-C_{a}^{k-1}(z)\left[I-\left(\frac{a_{k} \bar{a}_{k}-1}{a_{k}+1}\right)\left(\frac{z+1}{z \tilde{a}_{k}-1}\right) \bar{\eta}_{k} \eta_{k}^{T}\right] \\
& \times\left[C_{m}^{k-1}-\left(\frac{a_{k} \bar{a}_{k}-1}{\bar{a}_{k}+1}\right) \bar{\eta}_{k} \xi_{k}^{T}(A+I)\right] \\
= & \left(C-C_{a}^{k-1}(z) C_{m}^{k-1}\right)+C_{a}^{k-1}(z)\left[\left(\frac{a_{k} \bar{a}_{k}-1}{a_{k}+1}\right)\right.
\end{aligned}
$$




$$
\begin{aligned}
& \times\left(\frac{z+1}{z \bar{a}_{k}-1}\right) \bar{\eta}_{k} \eta_{k}^{T} C_{m}^{k-1}+\left(\frac{a_{k} \bar{a}_{k}-1}{\bar{a}_{k}+1}\right) \bar{\eta}_{k} \xi_{k}^{T}(A+I) \\
& \left.-\left(\frac{a_{k} \bar{a}_{k}-1}{a_{k}+1}\right)\left(\frac{z+1}{z \bar{a}_{k}-1}\right)\left(\frac{a_{k} \bar{a}_{k}-1}{\bar{a}_{k}+1}\right) \bar{\eta}_{k} \xi_{k}^{T}(A+I)\right] \\
= & \left(C-C_{a}^{k-1}(z) C_{m}^{k-1}\right)+\frac{\left(a_{k} \bar{a}_{k}-1\right)}{\left(z \bar{a}_{k}-1\right)\left(\bar{a}_{k}+1\right)\left(a_{k}+1\right)} C_{a}^{k-1}(z) \\
& \times\left[(z+1)\left(\bar{a}_{k}+1\right) \bar{\eta}_{k} \eta_{k}^{T} C_{m}^{k-1}+\left(z \bar{a}_{k}-1\right)\left(a_{k}+1\right) \bar{\eta}_{k} \xi_{k}^{T}\right. \\
& \left.\times(A+I)-(z+1)\left(a_{k} \bar{a}_{k}-1\right) \bar{\eta}_{k} \xi_{k}^{T}(A+I)\right] \\
= & \left(C-C_{a}^{k-1}(z) C_{m}^{k-1}\right) \\
& +\frac{\left(a_{k} \bar{a}_{k}-1\right)}{\left(z \bar{a}_{k}-1\right)\left(\bar{a}_{k}+1\right)\left(a_{k}+1\right)} C_{a}^{k-1}(z) \bar{\eta}_{k} \xi_{k}^{T} \\
& \times\left[(z+1)\left(\bar{a}_{k}+1\right)\left(a_{k} I-A\right)+\left(z \bar{a}_{k}-1\right)\left(a_{k}+1\right)\right. \\
& \left.\times(A+I)-(z+1)\left(a_{k} \bar{a}_{k}-1\right)(A+I)\right] \\
= & \left(C-C_{a}^{k-1}(z) C_{m}^{k-1}\right)+\frac{a_{k} \bar{a}_{k}-1}{z \bar{a}_{k}-1} C_{a}^{k-1}(z) \bar{\eta}_{k} \xi_{k}^{T}(z I-A) .
\end{aligned}
$$

In the above derivation we used the fact that $\eta_{k}^{T} C_{m}^{k-1}=\xi_{k}^{T}\left(a_{k} I-A\right)$. Now by definition of $E^{k}(z)$, we have

$$
\begin{aligned}
E^{k}(z)= & \left(C-C_{a}^{k}(z) C_{m}^{k}\right)(z I-A)^{-1} A K_{f} \\
= & \left(C-C_{a}^{k-1}(z) C_{m}^{k-1}\right)(z I-A)^{-1} A K_{f} \\
& +\frac{a_{k} \bar{a}_{k}-1}{z \bar{a}_{k}-1} C_{a}^{k-1}(z) \bar{\eta}_{k} \xi_{k}^{T} A K_{f} \\
= & E^{k-1}(z)+\frac{a_{k} \bar{a}_{k}-1}{z \bar{a}_{k}-1} C_{a}^{k-1}(z) \bar{\eta}_{k} \xi_{k}^{T} A K_{f} .
\end{aligned}
$$

Applying the above recursive formula and noting $E^{0}(z)=0$, we obtain the result (33).

\section{APPENDIX C: PROOF OF THEOREM 3.2}

From Theorem 3.1 we can see that it suffices to show that

$$
u^{T} E(z)=0, \forall u \in W^{\perp} .
$$

First, we would like to show by induction that the vector $\eta_{k}$ as defined in (26) lies in the subspace $W$ for $k=1,2, \ldots, l$.
For $k=1$, it is obvious since $\eta_{1}=w_{1}$ by definition. Suppose that it is true for $k=l-1$, i.e. $\eta_{k} \in W$ for $k=1,2, \ldots, l-1$. We need to show that it is also true for $k=l$. By Definition 2.2 we have that

$$
w_{l}^{T} C\left(a_{l} I-A\right)^{-1} B=w_{l}^{T} G\left(a_{l}\right)=0 .
$$

From Lemma 2.1 we know that

$$
G\left(a_{l}\right)=C_{a}^{l-1}\left(a_{l}\right) G_{m}^{l-1}\left(a_{l}\right),
$$

where $C_{a}^{l-1}(z)$ is as defined in Lemma 3.1. It follows from (C.1) and (C.2) that

$$
w_{l}^{T} C_{a}^{l-1}\left(a_{l}\right) G_{m}^{l-1}\left(a_{l}\right)=0 .
$$

By definition of $\eta_{l}$ (see (26)),

$$
\eta_{l}^{T} C_{m}^{l-1}\left(a_{l} l-A\right)^{-1} B=\eta_{l}^{T} G_{m}^{l-1}\left(a_{l}\right)=0 .
$$

Since $a_{l}$ is a distinct zero, the left nullspace of $G_{m}^{l-1}\left(a_{l}\right)$ is one-dimensional. Thus, (C.3) and (C.4) imply that there exists a constant $c_{l}$ such that

$$
\eta_{l}^{T}=c_{l} w_{l}^{T} C_{a}^{l-1}\left(a_{l}\right)
$$

Hence, we have

$$
\begin{aligned}
\eta_{l} & =c_{l} C_{a}^{l-1}\left(a_{l}\right)^{T} w_{l}, \\
& =c_{l}\left\{\prod_{k=1}^{l-1}\left[I-\left(\frac{a_{k} \bar{a}_{k}-1}{a_{k}+1}\right)\left(\frac{a_{l}+1}{a_{l} \bar{a}_{k}-1}\right) \eta_{k} \eta_{k}^{H}\right]\right\} w_{l} .
\end{aligned}
$$

After expanding the the right hand side of (C.5), one can see that $\eta_{l}$ lies in the span of $\left\{\eta_{1}, \eta_{2}, \ldots, \eta_{t-1}, w_{l}\right\}$ over $C^{m}$. Since $\eta_{1}, \eta_{2}, \ldots, \eta_{l-1}$ are in $W$ by assumption, we can conclude that $\eta_{1} \in W$.

Now we have established the fact that vectors $\eta_{1}, \eta_{2}, \ldots, \eta_{l}$ belong to the subspace $W$. Hence, if a vector is orthogonal to $W$, it must be orthogonal to $\eta_{1}, \eta_{2}, \ldots, \eta_{l}$.

From the above arguments, one can conclude that $u \in W^{\perp}$ implies that $u$ is orthogonal to $\eta_{1}, \eta_{2}, \ldots, \eta_{l}$. This fact together with Lemma 3.1 implies that

$$
u^{T} E(z)=0, \quad \forall u \in W^{\perp},
$$

which completes the proof. 